Artículo científico

Volumen 32(1):107-119. Enero-abril, 2021

e-ISSN 2215-3608, doi:10.15517/am.v32i1.40439

https://www.revistas.ucr.ac.cr/index.php/agromeso

\title{
Calidad de la proteína en líneas e híbridos experimentales de QPM. Altiplano Central, México ${ }^{1}$
}

\section{Protein quality in lines and experimental hybrids of QPM. Central Highlands, Mexico}

\author{
José Luis Arellano-Vázquez², Germán Fernando Gutiérrez-Hernández , Estela Flores-Gómez, \\ Daniel Eduardo López-Martínez ${ }^{3}$
}

1 Recepción: 20 de febrero, 2020. Aceptación: 8 de julio, 2020. Este trabajo fue resultado de la colaboración del Campo Experimental Valle de México del Instituto Nacional de Investigaciones Forestales, Agrícolas y Pecuarias y la Unidad Profesional Interdisciplinaria de Biotecnología del Instituto Politécnico Nacional.

2 Instituto Nacional de Investigaciones Forestales, Agrícolas y Pecuarias. Campo Experimental Valle de México. C. P. 56250. Coatlinchán, estado de México, México. arellano.jose@inifap.gob.mx (https://orcid.org/0000-0002-2231-2940).

3 Instituto Politécnico Nacional. Unidad Profesional Interdisciplinaria de Biotecnología. Av. Acueducto s/n. La Laguna Ticomán. C. P. 07340. Ciudad de México, México.gfgutierrez@ipn.mx (autor para la correspondencia: https://www.orcid.org/0000-0003-3312-3747), esfloresg@ ipn.mx (https://orcid.org/0000-0002-4634-455X), d.lopez.m@outlook.com (https://orcid.org/0000-0002-8642-2970).

\section{Resumen}

Introducción. Actualmente el maíz es el grano más cultivado en el mundo y es factible mejorar la nutrición de sus consumidores mediante el desarrollo del maíz con proteína de calidad (QPM, en inglés), cuya base es la expresión del gen opaco2, la cual incrementa los contenidos de lisina y triptófano del grano. Objetivo. Analizar químicamente la proteína del grano de líneas e híbridos simples experimentales de QPM y conocer las relaciones entre el genotipo y las variables evaluadas de la calidad proteica. Materiales y métodos. Los experimentos se efectuaron durante el 2017 en la Unidad Profesional Interdisciplinaria de Biotecnología del Instituto Politécnico Nacional (Ciudad de México). Se utilizaron granos enteros de líneas parentales (P1 a P6) y de los híbridos simples directos. Se estimaron lisina, triptófano, y cantidad y calidad de proteína. Se utilizó un diseño completamente al azar con tres repeticiones. Se hicieron análisis de varianza, comparación de medias y análisis de componentes principales. Resultados. Se identificaron como QPM significativos $(\mathrm{p} \leq 0,05)$ a las líneas $\mathrm{P}_{2}, \mathrm{P}_{3}$ y $\mathrm{P}_{6}$, y a los híbridos $\mathrm{P}_{2} \times \mathrm{P}_{4}$ y $\mathrm{P}_{2} \times \mathrm{P}_{6}$. El índice de calidad de proteína se correlacionó con el triptófano $\left(\mathrm{r}=0,9^{*}\right)$ y la lisina $\left(\mathrm{r}=0,7^{*}\right)$, pero no con la proteína total. Conclusiones. El factor fundamental para desarrollar los QPM fueron los aminoácidos esenciales y la limitante para incrementar su valor nutricional fue la calidad de proteína.

Palabras clave: aminoácidos esenciales, biofortificación, calidad proteica, opaco2.

\begin{abstract}
Introduction. Currently, maize is the most cultivated grain in the world and it is feasible to improve the nutrition of its consumers through the development of quality protein maize (QPM), whose basis is the expression of the opaque 2 gene, which increases the contents of lysine and tryptophan in the grain. Objective. To analyze chemically
\end{abstract}


the protein of the grain of lines and experimental simple hybrids of QPM and to know the relationships between the genotype and the evaluated variables of protein quality. Materials and methods. The experiments were carried out during 2017 in the Interdisciplinary Professional Unit of Biotechnology of the Instituto Politecnico Nacional (Mexico City). Whole grains of parental lines (P1 to P6) and direct simple hybrids were used. Lysine, tryptophan, and protein quantity and quality were estimated. A completely randomized design with three replications was used. Analysis of variance, comparison of means, and principal component analysis were performed. Results. Lines $\mathrm{P}_{2}, \mathrm{P}_{3}$, and $\mathrm{P}_{6}$, and hybrids $\mathrm{P}_{2} \times \mathrm{P}_{4}$ and $\mathrm{P}_{2} \times \mathrm{P}_{6}$ were identified as significant $(\mathrm{p} \leq 0.05)$ QPM. The protein quality index was correlated with tryptophan $(\mathrm{r}=0.9 *)$ and with lysine $(\mathrm{r}=0.7 *)$ but not with total protein. Conclusions. The fundamental factor for developing the QPM were the essential amino acids and the limiting factor for increasing their nutritional value was the quality of the protein.

Keywords: essential amino acids, biofortification, protein quality, opaque2.

\section{Introducción}

Actualmente, el maíz es el cultivo más producido en el mundo (1147 millones de toneladas de grano), en México se sembraron 7 millones de hectáreas y se cosecharon 27 millones de toneladas en el 2018 (Food and Agriculture Organization Statistics, 2020); este grano es fundamental en la alimentación de latinoamericanos, africanos subsaharianos y asiáticos (Njeri et al., 2017; Singh et al., 2020). El maíz es relevante económica, social y culturalmente (Fernández-Suárez et al., 2013), sobre todo para la población de recursos económicos escasos (Mageto et al., 2020).

El grano de maíz contiene las vitaminas niacina, $\beta$-caroteno (pro-vitamina A) y $\alpha$-tocoferol, y es rico en hidratos de carbono (58 al $72 \%$ ); pero su nivel de lípidos y proteínas es bajo (5 y $10 \%$, respectivamente) (López-Mazón et al., 2012) y sus proteínas son deficientes en triptófano y lisina (Serna-Saldívar et al., 2008; Singh et al., 2020), los cuales no se sintetizan en humanos (Ufaz \& Galili, 2008). Esta deficiencia nutrimental del grano convencional de maíz, puede repercutir en desnutrición, menoscabo del desarrollo corporal y cerebral, y susceptibilidad a enfermedades en personas cuya dieta se basa en este cultivo y es exigua en proteínas de origen animal (Singh et al., 2020).

La biofortificación consiste en incrementar la calidad nutritiva de los cultivos básicos per se, mediante mejoramiento genético y métodos de producción convencionales (Bouis et al., 2011), en esta estrategia está incluido el maíz con proteína de calidad (QPM, siglas en inglés), cuyos contenidos de lisina y triptófano en el grano duplican el del maíz común (no modificado) y por esta razón su calidad nutricional es elevada.

El gen opaco2 (o2) en condición homocigótica recesiva (o2o2), al expresarse reduce la síntesis de las zeínas, proteínas de almacenamiento del endospermo con contenido bajo de aminoácidos esenciales, e incrementa las no zeínas, que sí los contienen, por tanto, aumentan los aminoácidos esenciales del 60 al 100 \% (Ufaz \& Galili, 2008; Palacios-Rojas et al., 2017).

En la proteína del QPM la lisina está en el $4 \%$ de la secuencia primaria de las proteínas y el triptófano en el 0,8 $\%$, además, el nivel de este último es el $25 \%$ del de la lisina (Vivek et al., 2008), entre ambos aminoácidos existe una correlación alta $(0,9)$ (Palacios-Rojas et al., 2017). La proteína del QPM debe contener el doble de triptófano que el maíz normal cultivado en la misma región agrícola (Mendoza-Elos et al., 2006); sin embargo, hay autores (Vargas-Escobar et al., 2016) que aducen que la proporción de ese aminoácido en la proteína del grano entero debe ser del $0,075 \%$. Otro indicador del QPM es que la calidad de proteína (proporción entre triptófano y proteína total) debe ser mayor de 0,6 (Palacios-Rojas et al., 2017). El QPM actual posee endospermo duro y vítreo (Atlin 
et al., 2010), proteínas más digestibles (Paredes-López et al., 2009) y características de nixtamalización favorables (Serna-Saldívar et al., 2008; Vázquez-Carrillo et al., 2012; De-Groote et al., 2014).

Se considera que la evaluación conjunta de los factores genotípicos y químicos involucrados en la calidad de proteína del QPM, aportará indicadores útiles para el desarrollo de híbridos, cuya proteína del grano tenga un adecuado balance de aminoácidos esenciales, que puedan ser adoptados y cultivados por los productores de maíz del Altiplano Central de México.

El objetivo del presente trabajo fue analizar químicamente la proteína del grano de líneas e híbridos simples experimentales de QPM y conocer las relaciones entre el genotipo y las variables evaluadas de la calidad proteica.

\section{Materiales y métodos}

\section{Material genético}

Los experimentos reportados en el presente estudio se efectuaron durante el 2017 en la Unidad Profesional Interdisciplinaria de Biotecnología del Instituto Politécnico Nacional (Ciudad de México).

Se utilizaron seis líneas experimentales de maíz de grano con endospermo duro y color blanco cremoso $\left(\mathrm{P}_{1}\right.$ a $\left.\mathrm{P}_{6}\right)$, obtenidas del cruzamiento de las líneas CML $244\left(\mathrm{~S}_{4}\right)$, CML $352\left(\mathrm{~S}_{5}\right)$, CML $242\left(\mathrm{~S}_{4}\right), \mathrm{CML} 246\left(\mathrm{~S}_{3}\right)$, CML $349\left(\mathrm{~S}_{5}\right)$ y CML $354\left(\mathrm{~S}_{3}\right)$, con la fuente del opaco2 CML176 [(P63-12-2-1/P67-5-1-1)-1-2-B-B], desarrollada en el Centro Internacional de Mejoramiento de Maíz y Trigo (CIMMYT) y los híbridos simples directos (Cuadro 1). Se incluyeron tres testigos: maíz con proteína de calidad alta (QPM alto), maíz con proteína de calidad baja (QPM bajo) y maíz normal [H70, híbrido comercial cultivado en el Altiplano Central de México (2200 - 2600 msnm)].

Los genotipos parentales, los híbridos simples experimentales y el testigo H70, se cultivaron en el Campo Experimental Valle de México del Instituto Nacional de Investigaciones Forestales, Agrícolas y Pecuarias (Chapingo, México) en el 2016, con tres repeticiones, punta de riego y dosis de fertilización N 120- P 60- K 30 (kg ha $^{-1}$ ); mientras que el QPM alto y el QPM bajo se cultivaron el mismo año en el CIMMYT (Texcoco, México), en condiciones de riego y se fertilizaron con N 180- P 90- K $60\left(\mathrm{~kg} \mathrm{ha}^{-1}\right)$. En todos los casos, la cosecha fue manual y los granos permanecieron con contenido de humedad reducido (10 - 12\%) hasta la ejecución de los experimentos.

De la parcela cultivada se cosecharon diez plantas con competencia completa de cada tratamiento y repetición, las mazorcas se desgranaron manualmente, se mezclaron los granos de las repeticiones y se tomaron las muestras $(500 \mathrm{~g})$ al azar para efectuar las determinaciones químicas.

\section{Evaluación química de la proteína}

Se molieron 100 granos (completos) de cada genotipo (por repetición), se desgrasaron en equipo Soxhlet durante $8 \mathrm{~h}$, de esta harina se emplearon $100 \mathrm{mg}$ para hacer la cuantificación espectrofotométrica (Zuzi 4201/20, USA) de los aminoácidos. Para la lisina se utilizó la reacción del 2-cloro, 3, 5-dinitropiridina (390 nm) y para el triptófano la referencia fue el ácido glioxílico $(560 \mathrm{~nm})$ (Galicia et al., 2009). El nitrógeno total (\%) se estableció mediante el método del Technicon AutoAnalyzer II (Galicia et al., 2009) y se estimó la proteína total con la ecuación: Proteína $(\%)=\mathrm{N}_{2}(\%)(6,25)$ (Salinas-Moreno y Vázquez-Carrillo, 2006). La calidad de proteína se estimó por la proporción en que el triptófano estuvo presente en la proteína total del grano: calidad de proteína = [triptófano (\%) x 10 ${ }^{2}$ ] [proteína (\%)] $]^{-1}$ (Galicia et al., 2009). Los resultados se expresaron como porcentaje en base seca en promedio de tres repeticiones. 
Cuadro 1. Genotipos experimentales de QPM (líneas, híbridos simples experimentales y testigos) adaptados al Altiplano Central de México. Campo Experimental Valle de México del Instituto Nacional de Investigaciones Forestales, Agrícolas y Pecuarias. Chapingo, México, 2016.

Table 1. Experimental QPM genotypes (lines, experimental simple hybrids, and controls) adapted to the Central Highlands of Mexico. Valle de México Experimental Station. Instituto Nacional de Investigaciones Forestales, Agrícolas y Pecuarias. Chapingo, Mexico, 2016.

\begin{tabular}{|c|c|c|}
\hline Tratamiento & Descripción & Origen (2016) \\
\hline & Testigos & \\
\hline A & QPM bajo & CIMMYT, México \\
\hline $\mathrm{B}$ & QPM alto & CIMMYT, México \\
\hline \multirow[t]{2}{*}{$\mathrm{C}$} & H70 (Normal) & Chapingo, México \\
\hline & Líneas & \\
\hline $\mathrm{D}$ & $\mathrm{P}_{1}$ & Chapingo, México \\
\hline $\mathrm{E}$ & $\mathrm{P}_{2}$ & Chapingo, México \\
\hline $\mathrm{F}$ & $\mathrm{P}_{3}$ & Chapingo, México \\
\hline G & $\mathrm{P}_{4}$ & Chapingo, México \\
\hline $\mathrm{H}$ & $\mathrm{P}_{5}$ & Chapingo, México \\
\hline \multirow[t]{2}{*}{ I } & $\mathrm{P}_{6}$ & Chapingo, México \\
\hline & Híbridos experimentales & \\
\hline $\mathrm{J}$ & $\mathrm{P}_{1} \times \mathrm{P}_{2}$ & Chapingo, México \\
\hline $\mathrm{K}$ & $\mathrm{P}_{1} \times \mathrm{P}_{3}$ & Chapingo, México \\
\hline $\mathrm{L}$ & $\mathrm{P}_{1} \times \mathrm{P}_{4}$ & Chapingo, México \\
\hline M & $\mathrm{P}_{1} \times \mathrm{P}_{5}$ & Chapingo, México \\
\hline $\mathrm{N}$ & $\mathrm{P}_{1} \times \mathrm{P}_{6}$ & Chapingo, México \\
\hline $\mathrm{O}$ & $\mathrm{P}_{2} \times \mathrm{P}_{3}$ & Chapingo, México \\
\hline $\mathrm{P}$ & $\mathrm{P}_{2} \times \mathrm{P}_{4}$ & Chapingo, México \\
\hline Q & $\mathrm{P}_{2} \times \mathrm{P}_{5}$ & Chapingo, México \\
\hline $\mathrm{R}$ & $\mathrm{P}_{2} \times \mathrm{P}_{6}$ & Chapingo, México \\
\hline $\mathrm{S}$ & $\mathrm{P}_{3} \times \mathrm{P}_{4}$ & Chapingo, México \\
\hline $\mathrm{T}$ & $\mathrm{P}_{3} \times \mathrm{P}_{5}$ & Chapingo, México \\
\hline $\mathrm{U}$ & $\mathrm{P}_{3} \times \mathrm{P}_{6}$ & Chapingo, México \\
\hline $\mathrm{V}$ & $\mathrm{P}_{4} \times \mathrm{P}_{5}$ & Chapingo, México \\
\hline W & $\mathrm{P}_{4} \times \mathrm{P}_{6}$ & Chapingo, México \\
\hline $\mathrm{X}$ & $\mathrm{P}_{5} \times \mathrm{P}_{6}$ & Chapingo, México \\
\hline
\end{tabular}

\section{Análisis estadístico}

Se utilizó un diseño completamente al azar con tres repeticiones de 100 granos. Se hicieron análisis de varianza, comparación de medias con la prueba de Tukey $(\mathrm{p} \leq 0,05)$, prueba de correlación de Pearson $(\mathrm{p} \leq 0,05)$ y análisis de componentes principales. Para la prueba de Tukey, los datos porcentuales se transformaron con la función arcoseno $\sqrt{ } \mathrm{x}$ para ajustarlos a una distribución normal. Para determinar si el procedimiento de análisis de componentes principales era adecuado para el presente estudio, se utilizó el criterio de la varianza total explicada (Peña, 2002), para lo cual se calcularon los autovectores de la matriz de correlación. El procesamiento estadístico de los datos se hizo con el programa SAS (SAS Institute Inc., version 9.0). 


\section{Resultados}

Hubo diferencias significativas ( $\leq 0,01$ ) entre tratamientos (genotipos) para todas las cuantificaciones químicas (Cuadro 2), con coeficientes de variación reducidos, a excepción de lisina. El modelo experimental empleado fue confiable ( $\mathrm{R}^{2}$ del 75 al $98 \%$ ). Con base en lo anterior, se efectuó la comparación de los valores medios de los tratamientos con la prueba de Tukey $(\mathrm{p} \leq 0,05)$ (Figuras 1 y 2 ).

Cuadro 2. Análisis de varianza de la evaluación química de la proteína de las líneas parentales de QPM e híbridos simples experimentales adaptados al Altiplano Central de México. Unidad Profesional Interdisciplinaria de Biotecnología del Instituto Politécnico Nacional. Ciudad de México, México, 2017.

Table 2. Analysis of variance of the chemical evaluation of the protein of the QPM parental lines and experimental simple hybrids adapted to the Central Highlands of Mexico. Interdisciplinary Professional Unit of Biotechnology, Instituto Politécnico Nacional. Mexico City, Mexico, 2017.

\begin{tabular}{cccccc}
\hline Variable & C. V. $(\boldsymbol{\%})$ & $\mathbf{R}^{\mathbf{2}}(\boldsymbol{\%})$ & Cuadrado Medio & Error & \\
\hline Triptófano & 5,9 & 98 & $1,1\left(10^{-3}\right)$ & $2,5\left(10^{-5}\right)$ & $* *$ \\
Lisina & 20,3 & 75 & $15,6\left(10^{-3}\right)$ & $5,1\left(10^{-3}\right)$ & $* *$ \\
Proteína & 2,1 & 98 & 2,7 & $76,7\left(10^{-3}\right)$ & $* *$ \\
Calidad de proteína & 5,8 & 97 & $52,9\left(10^{-3}\right)$ & $1,4\left(10^{-3}\right)$ & $* *$ \\
\hline
\end{tabular}

**: altamente significativo $(\mathrm{p} \leq 0,01), \mathrm{C}$. V.: coeficiente de variación, $\mathrm{R}^{2}$ : coeficiente de determinación / **: highly significant ( $\left.\mathrm{p} \leq 0.01\right)$, C. V.: coefficient of variation, $\mathrm{R}^{2}$ : coefficient of determination.

Los testigos QPM bajo y H70, tuvieron significativamente $(\mathrm{p} \leq 0,05)$ el contenido menor de aminoácidos y de cantidad y calidad de proteína. En contraste, el testigo QPM alto mostró los valores mayores $(\mathrm{p} \leq 0,05)$ en esas variables, excepto proteína, inclusive su calidad proteica fue la más alta.

Las líneas E, F, H e I expresaron los contenidos más altos $(\mathrm{p} \leq 0,05)$ de triptófano y/o lisina (Figura 1). Los híbridos simples significativos en uno o ambos aminoácidos fueron $\mathrm{O}, \mathrm{P}, \mathrm{Q}, \mathrm{R}, \mathrm{T}, \mathrm{U}, \mathrm{W}$ y X (Figura 1) y en proteína total J, O, P, Q, R y U; la calidad de proteína superior $(\mathrm{p} \leq 0,05)$ correspondió al QPM alto, seguido de las líneas E, F e I y los híbridos P y R (Figura 2). Sobresalió que todos los híbridos mencionados ocuparon el mismo nivel significativo que uno u otro de sus progenitores en las variables señaladas.

En el análisis de correlación (Cuadro 3), triptófano y lisina se asociaron significativamente entre sí, con la proteína total y con la calidad de proteína, y esta no se relacionó con la proteína total. Gráficamente (Figura 3), se corroboró que la cantidad de proteína del H70 (testigo regional) fue de las más reducidas $(\mathrm{p} \leq 0,05)$ de los tratamientos (Figura 2) y la calidad de proteína fue independiente de la proteína total, pero sí se vinculó con el triptófano, sobre todo en los tratamientos B, H, I, M, y W.

El análisis de componentes principales realizado (Cuadro 4) fue pertinente dado que los primeros dos componentes calculados explicaron el $98 \%$ de la varianza de los datos experimentales.

El componente principal 1 aglutinó el $76 \%$ de la varianza experimental y, por la magnitud de sus vectores propios, para la formación del QPM contribuyeron primordialmente las cantidades de triptófano, lisina y, en proporción menor, la calidad de proteína; mientras que en el componente 2, que explicó el $22 \%$ restante de la variación, solamente sobresalió la cantidad de proteína por su valor alto y positivo (Cuadro 5). 


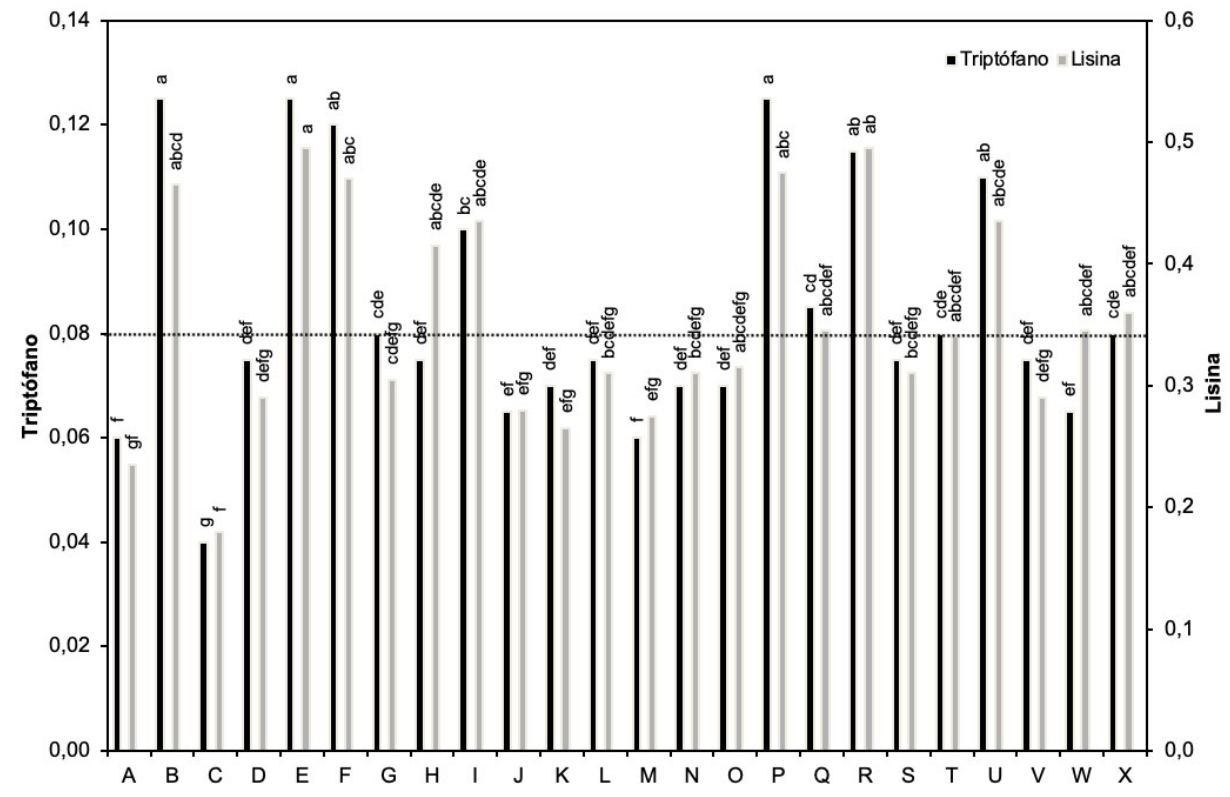

Figura 1. Valores medios (\%) de triptófano y lisina de líneas parentales de QPM e híbridos simples experimentales adaptados al Altiplano Central de México. Unidad Profesional Interdisciplinaria de Biotecnología del Instituto Politécnico Nacional. Ciudad de México, México, 2017.

Medias con la misma letra en cada genotipo y variable fueron significativamente iguales $(\mathrm{p} \leq 0,05)$. Línea punteada: umbral de triptófano en el QPM.

Figure 1. Mean values (\%) of tryptophan and lysine of QPM parental lines and experimental simple hybrids adapted to the Central Highlands of Mexico. Interdisciplinary Professional Unit of Biotechnology, Instituto Politécnico Nacional. Mexico City, Mexico, 2017.

Means with the same letter in each genotype and variable were significantly equal $(\mathrm{p} \leq 0.05)$. Dotted line: tryptophan threshold in the QPM.

En la representación bidimensional (Figura 4) de los genotipos experimentales y las variables químicas evaluadas, se advirtió que la cantidad de proteína se localizó en el cuadrante I del plano cartesiano, así que se asoció positivamente con ambos componentes principales, sobre todo al 2 porque su vector quedo más cercano a él.

Los genotipos E, Q, R, T y U compartieron el cuadrante I, el segundo de ellos quedó contiguo a la proteína, y E y R a los aminoácidos y con afinidad mayor al componente 1. La mitad (54\%) de los genotipos bajo estudio se alojó en los cuadrantes II y III, en el primero de ellos se asociaron positivamente al componente 1 y negativamente al 2 y, en el segundo, la vinculación fue negativa con ambos, en esta situación se encontraron los testigos A (QPM bajo) y C (H70).

Lisina, triptófano y calidad de proteína, se ubicaron en el cuadrante IV, por lo que se relacionaron positivamente al componente 1 y negativamente al componente 2, aunque fueron más cercanos al primero de ellos; también quedaron próximos a los genotipos F y P, así como distantes de I y más aún de B (QPM alto). Contrastó la pendiente de los vectores de los aminoácidos, muy cercanos al componente 1 , con la de calidad proteica, que fue intermedia entre ambos componentes. 


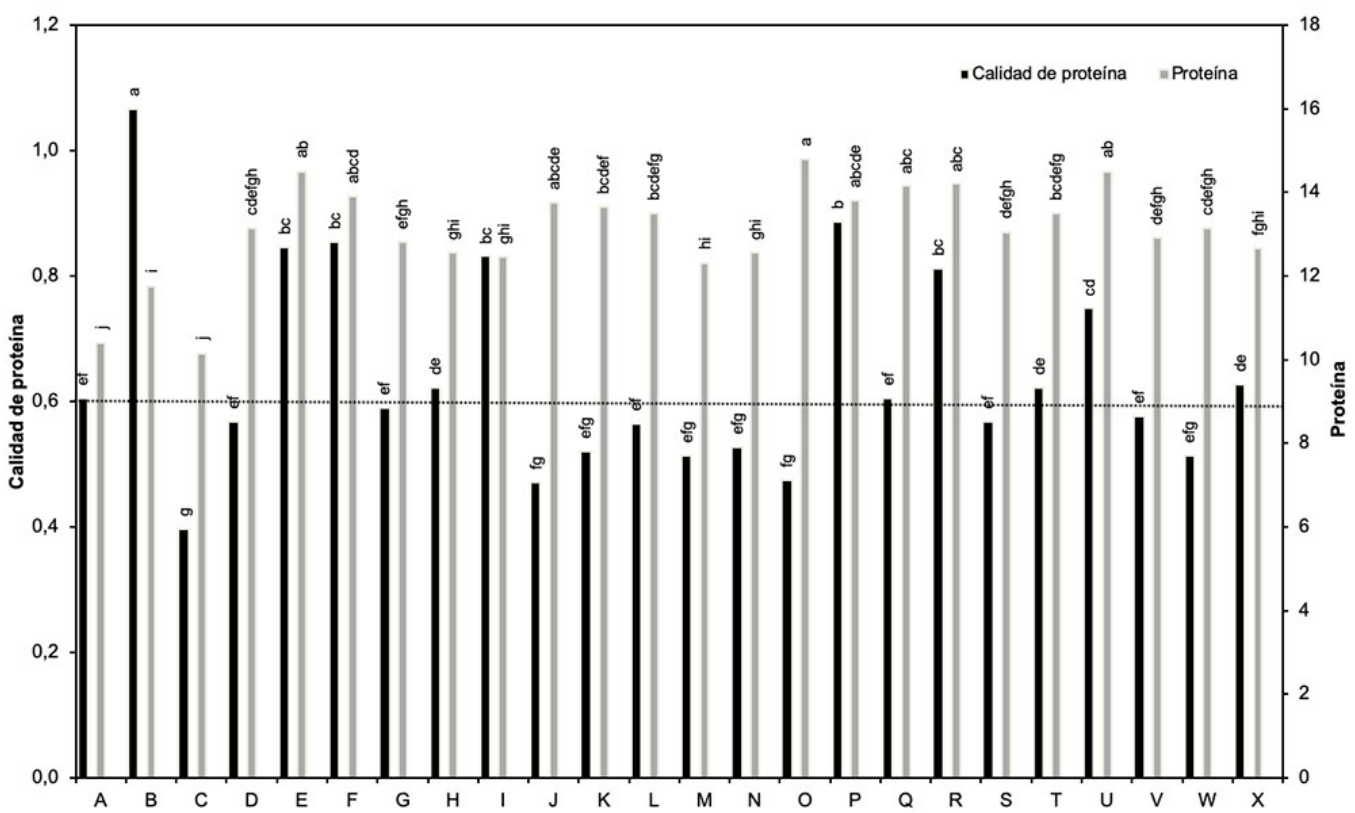

Figura 2. Valores medios de la calidad de proteína y de la proteína total (\%) de líneas parentales de QPM e híbridos simples experimentales adaptados al Altiplano Central de México. Unidad Profesional Interdisciplinaria de Biotecnología del Instituto Politécnico Nacional. Ciudad de México, México, 2017.

Medias con la misma letra en cada genotipo y variable fueron significativamente iguales $(\mathrm{p} \leq 0,05)$. Línea punteada: umbral de calidad de proteína en el QPM.

Figure 2. Mean values of protein quality and total protein (\%) of QPM parental lines and experimental simple hybrids adapted to the Central Highlands of Mexico. Interdisciplinary Professional Unit of Biotechnology, Instituto Politécnico Nacional. Mexico City, Mexico, 2017.

Means with the same letter in each genotype and variable were significantly equal $(\mathrm{p} \leq 0.05)$. Dotted line: protein quality threshold in the QPM.

Cuadro 3. Análisis de correlación entre las variables químicas de la proteína de líneas parentales de QPM e híbridos simples experimentales adaptados al Altiplano Central de México. Unidad Profesional Interdisciplinaria de Biotecnología del Instituto Politécnico Nacional. Ciudad de México, México, 2017.

Table 3. Correlation analysis between the chemical variables of the protein of QPM parental lines and experimental simple hybrids adapted to the Central Highlands of Mexico. Interdisciplinary Professional Unit of Biotechnology, Instituto Politécnico Nacional. Mexico City, Mexico, 2017.

\begin{tabular}{ccccc}
\hline & Triptófano & Lisina & Proteína & Calidad de proteína \\
\hline Triptófano & 1,0 & $0,8^{*}$ & $0,5^{*}$ & $0,9^{*}$ \\
Lisina & & 1,0 & $0,5^{*}$ & $0,7^{*}$ \\
Proteína & & 1,0 & $0,2 \mathrm{~ns}$ \\
Calidad de proteína & & & 1,0 \\
\hline
\end{tabular}

*: significativo ( $\mathrm{p} \leq 0,05)$; ns: no significativo. / *: significant ( $\mathrm{p} \leq 0.05)$; ns: not significant. 

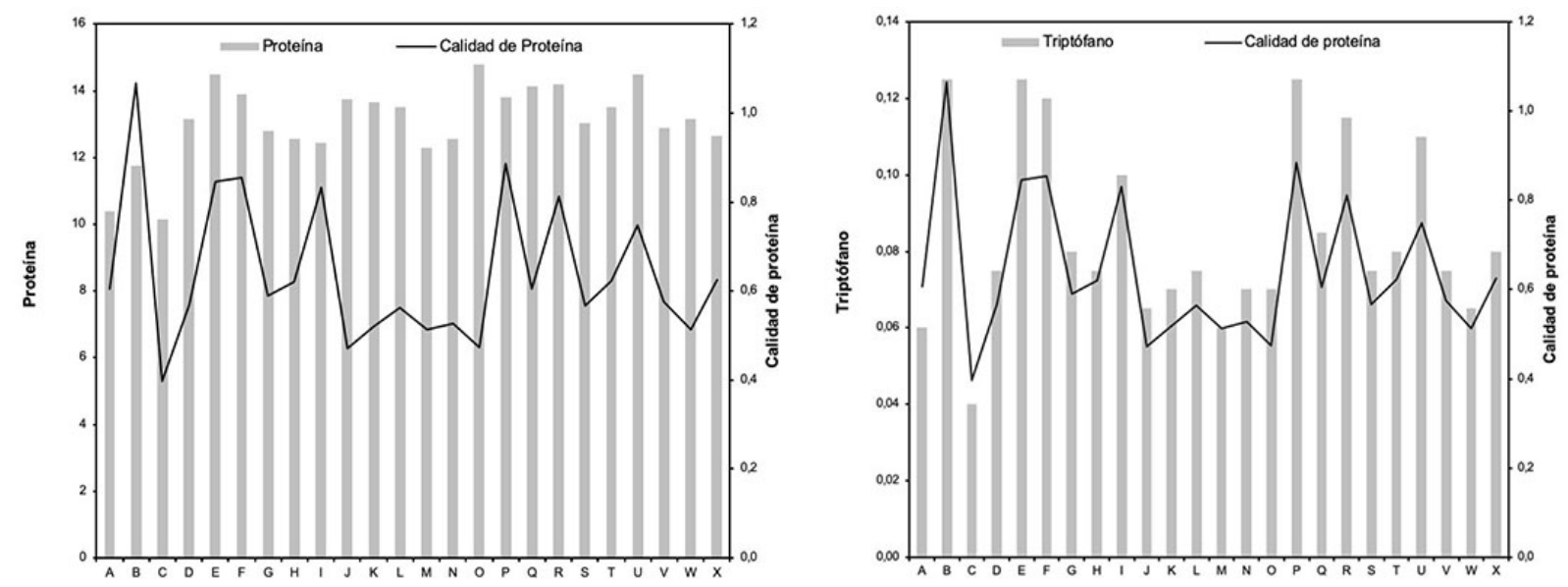

Figura 3. Fluctuación de la calidad de proteína y de los contenidos de proteína y triptófano en líneas parentales de QPM e híbridos simples experimentales adaptados al Altiplano Central de México. Unidad Profesional Interdisciplinaria de Biotecnología del Instituto Politécnico Nacional. Ciudad de México, México, 2017.

Figure 3. Fluctuation of protein quality and protein and tryptophan contents of QPM parental lines and experimental simple hybrids adapted to the Central Highlands of Mexico. Interdisciplinary Professional Unit of Biotechnology, Instituto Politécnico Nacional. Mexico City, Mexico, 2017.

Cuadro 4. Valores propios y varianza explicada (\%) del análisis de componentes principales realizado para las variables químicas de la proteína de líneas parentales de QPM e híbridos simples experimentales adaptados al Altiplano Central de México. Unidad Profesional Interdisciplinaria de Biotecnología del Instituto Politécnico Nacional. Ciudad de México, México, 2017.

Table 4. Eigenvalues and explained variance (\%) of the principal component analysis performed for the chemical variables of the protein of QPM parental lines and experimental simple hybrids adapted to the Central Highlands of Mexico. Interdisciplinary Professional Unit of Biotechnology, Instituto Politécnico Nacional. Mexico City, Mexico, 2017.

\begin{tabular}{cccc}
\hline Componente & Autovalor & Varianza & Acumulada \\
\hline 1 & 3,03 & 76 & 76 \\
2 & 0,87 & 22 & 98 \\
3 & 0,07 & 2 & 99 \\
4 & 0,02 & 1 & 0 \\
\hline
\end{tabular}

Cuadro 5. Vectores propios de los componentes principales 1 y 2 de las variables químicas de la proteína de líneas parentales de QPM e híbridos simples experimentales adaptados al Altiplano Central de México. Unidad Profesional Interdisciplinaria de Biotecnología del Instituto Politécnico Nacional. Ciudad de México, México, 2017.

Table 5. Eigenvectors of the main components 1 and 2 of the chemical variables of the protein of QPM parental lines and experimental simple hybrids adapted to the Central Highlands of Mexico. Interdisciplinary Professional Unit of Biotechnology, Instituto Politécnico Nacional. Mexico City, Mexico, 2017.

\begin{tabular}{ccc}
\hline Variable & Componente 1 & Componente 2 \\
\hline Triptófano & 0,5665 & $-0,5770$ \\
Lisina & 0,5585 & $-0,0429$ \\
Proteína total & 0,3085 & 0,9023 \\
Calidad de proteína & 0,5216 & $-0,4251$ \\
\hline
\end{tabular}




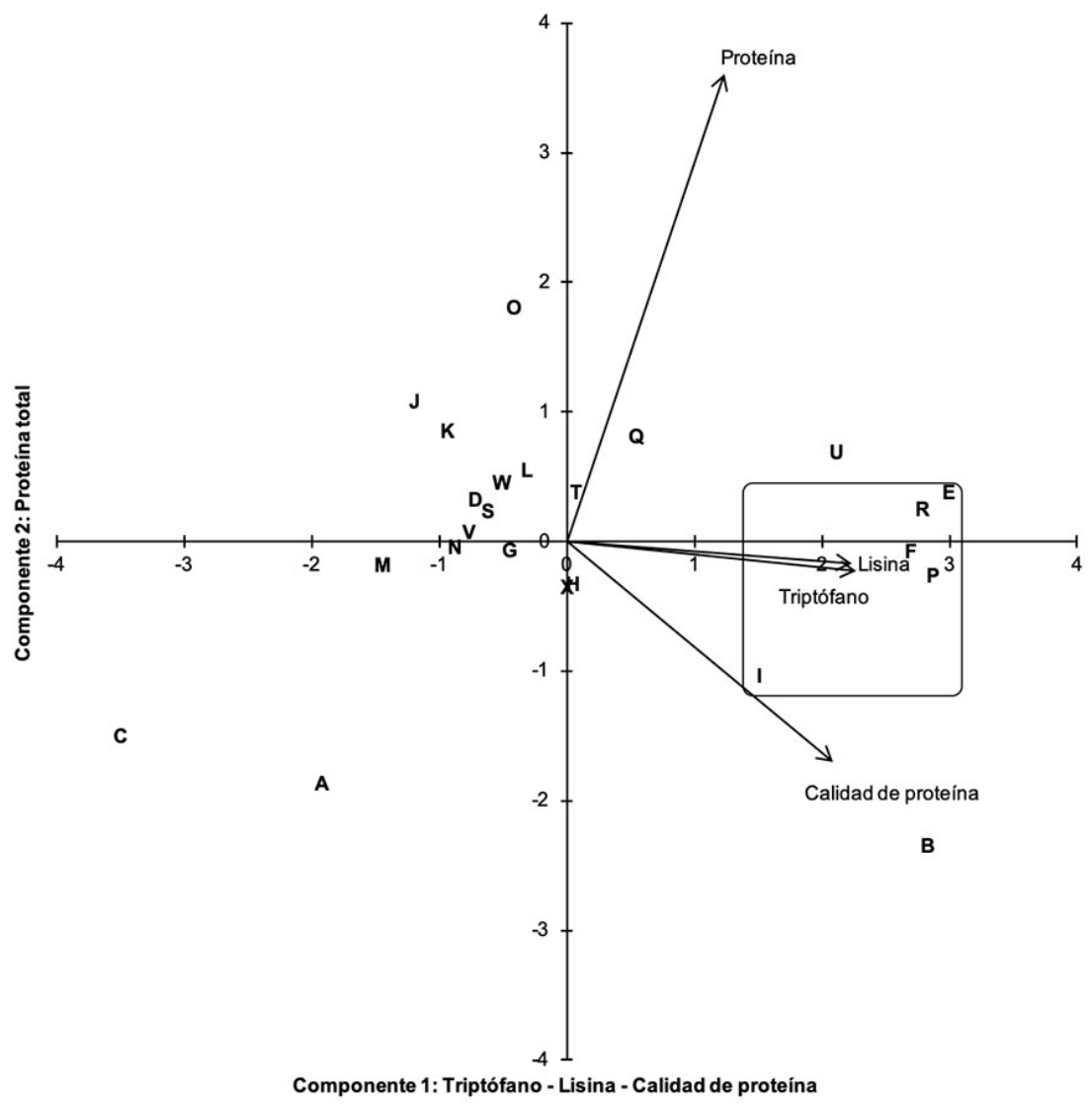

Figura 4. Representación bidimensional (24 genotipos y 4 variables químicas evaluadas) de los componentes principales 1 y 2 obtenidos en líneas parentales de QPM e híbridos simples experimentales adaptados al Altiplano Central de México. Unidad Profesional Interdisciplinaria de Biotecnología del Instituto Politécnico Nacional. Ciudad de México, México, 2017.

Figure 4. Two-dimensional representation (24 genotypes and 4 chemical variables evaluated) of the main components 1 and 2 obtained in QPM parental lines and experimental simple hybrids adapted to the Central Highlands of Mexico. Interdisciplinary Professional Unit of Biotechnology Instituto Politécnico Nacional. Mexico City, Mexico, 2017.

\section{Discusión}

Los genotipos experimentales de QPM fueron diferentes $(\mathrm{p} \leq 0,01)$ en todas las variables químicas de la proteína del grano (Cuadro 2), esto indicó que las líneas y los híbridos simples formados con ellas fueron distintos entre sí. Las líneas parentales bajo estudio, disímiles en genotipo y grado de endogamia, fueron receptoras de la misma fuente del opaco2; en consecuencia, dicho gen se expresó en un contexto genético y metabólico particular, cada línea modificada lo integró a su genoma de manera específica y se produjeron híbridos simples de QPM que manifestaron atributos heterogéneos de calidad de proteína, con variaciones causadas por la recombinación genética inherente a la cruza y, probablemente, por la participación de genes reguladores de la síntesis de lisina y triptófano en el endospermo del grano (Palacios-Rojas et al., 2017). 
En comparación con el H70, maíz no modificado, las líneas y los híbridos expresaron cantidades significativas $(\mathrm{p} \leq 0,05)$ de ambos aminoácidos esenciales en la proteína del grano, esto sería consecuencia de la expresión del opaco2 (Sofi et al., 2009), que redujo las proteínas deficientes en lisina y triptófano (zeínas) y aumentó las que tienen proporción alta de esos aminoácidos esenciales (no zeínicas) (Serna-Saldívar et al., 2008; Mansilla et al., 2019). Todos los genotipos modificados superaron $(\mathrm{p} \leq 0,05)$ en proteína al H70, esto sugiere que la integración de los genotipos experimentales de QPM inició con la modificación de líneas con cantidad de proteína elevada (Palacios-Rojas et al., 2017).

En todos los tratamientos, el contenido de triptófano fue mayor que el de lisina, lo cual fue establecido también por Vivek et al. (2008). El testigo regional (H70) tuvo 0,04 \% de triptófano en la proteína total (Figura 1), así que, de acuerdo con Mendoza-Elos et al. (2006), el contenido mínimo de este aminoácido para dictaminar un QPM en el presente estudio fue de 0,08\%. Los genotipos que cumplieron esta condición fueron el testigo QPM alto, las líneas E, F, G e I, y los híbridos P, Q, R, T, U y X; los cuales también tuvieron una calidad proteica mínima de 0,6 para ser QPM (Palacios-Rojas et al., 2017), el QPM bajo y la línea H cubrieron también este último requisito.

$\mathrm{Al}$ considerar la significancia $(\mathrm{p} \leq 0,05)$ de los tratamientos clasificados como QPM, los mejores en triptófano fueron B, E, F, P, R y U, y en calidad proteica solo el B alcanzó el primer nivel significativo; mientras que en el segundo estuvieron E, F, I, P y R. Este resultado podría ser consecuencia de que el QPM alto se cultivó con una dosis de fertilización mayor y esto pudo incidir en la calidad de proteína del grano producido, aunque existe controversia sobre esto (Wegary et al., 2011). La superioridad del testigo QPM alto denotó también que existe aún potencial para aumentar el valor nutricional de los QPM, a través del manejo agronómico del cultivo, cambiando las líneas modificadas con el opaco2 o la fuente de este.

Los híbridos experimentales expresaron niveles de triptófano, lisina, proteína y calidad de proteína análogos a los de sus correspondientes progenitores (Figuras 1 y 2), las variaciones que presentaron se pueden atribuir a la recombinación genética ocurrida (Singh et al., 2020) y al efecto de genes modificadores de la cantidad de los aminoácidos esenciales en el grano de maíz (Palacios-Rojas et al., 2017).

Las líneas E, F e I mostraron consistentemente los contenidos mayores ( $\mathrm{p} \leq 0,05)$ de triptófano, lisina, proteína y calidad de proteína; además, fueron progenitoras de los híbridos clasificados como QPM por contenido de triptófano y por calidad proteica $(\mathrm{P}, \mathrm{Q}, \mathrm{R}, \mathrm{T}, \mathrm{U}$ y X). Sobresalió la línea E, una de las más homocigóticas del grupo, porque participó como hembra en los híbridos $\mathrm{P}$ y $\mathrm{R}$, cuyos valores de ambos aminoácidos y cantidad y calidad de proteína, fueron los mejores desde el punto de vista estadístico.

La lisina y el triptófano se correlacionaron de manera significativa $(\mathrm{p} \leq 0,05)$ lo que coincide con Njeri et al. (2017) (Cuadro 3), esto permitiría estimar la calidad de proteína con referencia a cualquiera de los aminoácidos; no obstante, en el presente estudio se hizo con base al triptófano, debido a que tuvo un coeficiente de variación 3,5 veces menor que el de lisina (Cuadro 2) y a que el método es más sencillo y de menor costo (Njeri et al., 2017).

Las variables primordiales del componente 1 fueron triptófano y lisina (en orden descendente), en el componente 2 solo destacó la cantidad de proteína (Cuadro 5), esto expuso que para obtener el QPM primero se requirieron los aminoácidos y, después, que estos se articulen en la secuencia de aminoácidos de las proteínas.

Las fluctuaciones de la cantidad y la cantidad de proteína, y del triptófano entre tratamientos (Figura 3), indicaron que la primera variable, aunque de inicio debe ser alta (Palacios-Rojas et al., 2017), no intervino para fijar la calidad nutricional de la proteína, como sí lo hizo el triptófano. Esto independientemente de que la calidad proteica se calculó con base en el triptófano, ya que pudieron quedar residuos libres de este aminoácido que no se ensamblaron en la secuencia de aminoácidos de las proteínas del grano (Mansilla et al., 2019), por eso la correlación no fue perfecta. Esto motivó diferencias de calidad proteica entre los genotipos analizados, a las cuales se sumaron la variación causada por la recombinación genética entre las líneas y la regulación genética del contenido de aminoácidos esenciales. 
En el análisis de componentes principales (Figura 4) se visualizaron en el plano cartesiano a los genotipos experimentales y a los vectores de las variables químicas evaluadas. El vector del contenido proteico se ubicó en el cuadrante 1, cercano al componente 2 y con una pendiente alta, así que esta variable se incrementó rápidamente al variar ambos componentes, es decir, fue el aspecto químico que se favoreció en el conjunto de las combinaciones de los niveles de las variables químicas analizadas. La línea E y los híbridos Q, R, T y U se localizaron en el cuadrante I, esto es, respondieron mejor para proteína total.

En contraste, en el cuadrante IV se ubicaron los genotipos de los cuales fue más complicado obtener las cantidades de los aminoácidos y la calidad de proteína, es decir, aumentaron con el componente 1 pero disminuyeron con el 2; este comportamiento se agudizó para conseguir la calidad proteica, dado que esta disminuyó concomitantemente con el incremento de lisina y triptófano, situación que confirmó que no todos los aminoácidos se incorporaron a la secuencia de aminoácidos de las proteínas del grano, esto se advirtió también en la fluctuación del triptófano y la calidad de proteína a través de los genotipos (Figura 3). En consecuencia, la obtención de QPM implica propiciar las combinaciones genéticas mediante las cuales se aumenten los niveles de aminoácidos esenciales y que gran parte de estos se ensamblen a las proteínas, para esto es preciso hacer la evaluación química de la proteína y retroalimentar la labor del fitomejorador.

Los tratamientos de respuesta mayor para calidad de proteína (Figura 2) estuvieron en los cuadrantes I y IV, de los cuales las líneas E, F e I, y los híbridos simples P y R (recuadro de la Figura 4), fueron los significativos $(\mathrm{p} \leq 0,05)$, variaron positivamente con los aminoácidos y la calidad proteica (componente 1) y modificaron escasamente su respuesta (negativa o positiva) a los cambios en la cantidad de proteína (componente 2). En los cuadrantes II y III se alojaron la mayoría de los genotipos bajo estudio, ninguno de ellos alcanzó el contenido de aminoácidos esenciales ni la calidad proteica de las líneas y los híbridos destacados.

La línea I y el testigo QPM alto (B) se alinearon con el vector de la calidad de proteína, ambos genotipos fueron QPM prominentes; sin embargo, la distancia entre ambos tratamientos fue amplia, esto corrobora que aún existe potencial para mejorar el QPM destinado al Altiplano Central de México.

Para dictaminar un QPM como superior es preciso considerar en su conjunto las propiedades químicas de la proteína, enfatizando la calidad proteica sin menoscabo de los aminoácidos e involucrar el desempeño agronómico; este aspecto se aborda actualmente en el Programa de Maíz del Campo Agrícola Experimental Valle de México, Instituto Nacional de Investigaciones Forestales, Agrícolas y Pecuarias (México).

\section{Conclusiones}

Las proporciones de lisina, triptófano, y cantidad y calidad de proteína se establecieron y expresaron diferencialmente en cada línea parental modificada con el opaco2, esos valores se mantuvieron en los híbridos simples directos formados con ellas. El triptófano determinó primordialmente la calidad de proteína del grano del QPM y el factor limitante de la calidad nutricional fue también la calidad de proteína.

\section{Agradecimientos}

Los autores extienden su gratitud al Programa de Maíz del Campo Experimental Valle de México (CEVAMEX, INIFAP), al Instituto Politécnico Nacional (SIP: 20190236) y a la Comisión de Operación y Fomento de Actividades Académicas del IPN. 


\section{Referencias}

Atlin, G. N., Palacios-Rojas, N., Babu, R., Twumasi-Afriye, S., De Groote, H., Vivek, B., Friesen, D., \& Pixley, K. V. (2010). Quality protein maize: progress, impact, and prospects. In J. Janick (Ed.), Plant Breeding Reviews, (83-130, Vol 34). Wiley \& Sons, Inc. https://doi.org/10.1002/9780470880579.ch3

Bouis,H.E.,Hotz,C.,McClafferty,B.,Meenakshi,J.V.,\&Pfeiffer,W.H.(2011).Biofortification:Anew tool to reduce micronutrient malnutrition. Food \& Nutrition Bulletin, 32(1 Suppl), 31S-40S. https://doi.org/10.1177/15648265110321S105.

De-Groote, H., Gunaratna, N. S., Okuro, J. O., Wondimu, A., Chege, C. K., \& Tomlins, K. (2014). Consumer acceptance of quality protein maize (QPM) in East Africa. Journal of the Science of Food and Agriculture, 94(15), 3201-3212. https://doi.org/10.1002/jsfa.6672

Fernández-Suárez, R., Morales-Chávez, L. A., \& Gálvez-Mariscal, A. (2013). Importancia de los maíces nativos de México en la dieta nacional. Una revisión indispensable. Revista Fitotecnia Mexicana, 36(suplemento 3 A), 275-283.

Food and Agriculture Organization Statistics. (2020). Food and agriculture data. Retrieved May 19, 2020, from https://www. fao.org/faostat/es/\#data/QC

Galicia, L., Nurit, E., Rosales, A., \& Palacios-Rojas, N. (2009). Laboratory protocols: Maize nutrition quality and plant tissue analysis laboratory. International Maize and Wheat Improvement Center.

López-Mazón, S. L., García-Navarrete, G., \& Ibarra-Gutiérrez, B. N. (2012). El maíz (Zea mays L.) y la cultura Maya. BIOtecnia, 14(3), 3-8. https://doi.org/10.18633/bt.v14i3.123

Mageto, E. K., Lee, M., Dhliwayo, T., Palacios-Rojas, N., San-Vicente, F., Burgueño, J., \& Hallauer, A. R. (2020). An evaluation of kernel zinc in hybrids of elite quality protein maize (QPM) and non-QPM inbred lines adapted to the tropics based on a mating design. Agronomy, 10(5), 695. https://doi.org/10.3390/agronomy10050695

Mansilla, P. S., Nazar, M. C., \& Pérez, G. T. (2019). Evaluación y comparación de la composición y calidad proteica de familias de medios hermanos de maíz opaco-2 (Zea mays L.) de Argentina. AgriScientia, 36(1), 39-53. https://doi. org/10.31047/1668.298x.v36.n1.22682

Mendoza-Elos, M., Andrio-Enríquez, E., Juárez-Goiz, J. M., Mosqueda-Villagómez, C., Latournerie-Moreno, L., CastañónNájera, G., López-Benítez, A., \& Moreno-Martínez, E. (2006). Contenido de lisina y triptófano en genotipos de maíz de alta calidad proteica y normal. Revista Universidad y Ciencia, 22(2), 153-161.

Njeri, S. G., Makumbi, D., Warburton, M. L., Diallo, A., Jumbo, M. B., \& Chemining'wa, G. (2017). Genetic analysis of tropical quality protein maize (Zea mays L.) germplasm. Euphytica, 213, 261 . https://doi.org/10.1007/s10681-017-2048-4

Palacios-Rojas, N., Twumasi-Afriyie, S., Friesen, D., Teklewold, A., Wegary, D., De Groote, H., Rosales, A., Narro-León, L., Chassaigne, A., Padilla, R., Vargas-Escobar, E. A., López, K., Bowen, C., \& Prasanna, B. M. (2017). Lineamientos para el control de calidad de semilla y grano de maíz de alta calidad proteica (QPM): Experiencia en el desarrollo y promoción de QPM en Latinoamérica. CIMMYT.

Paredes-López, O., Guevara, L. F., \& Bello Pérez, L. A. (2009). La nixtamalización y el valor nutritivo del maíz. Ciencias, 92-93, 60-70.

Peña, D. (2002). Análisis de datos multivariantes. McGraw-Hill Interamericana. 
Salinas-Moreno, Y., \& Vázquez-Carrillo, G. (2006). Metodologías de análisis de la calidad nixtamalera-tortillera en maíz. Instituto Nacional de Investigaciones Forestales Agrícolas y Pecuarias.

Serna-Saldívar, S. O., Amaya-Guerra, C. A., Herrera-Macías, P., Melesio-Cuéllar, J. L., Preciado-Ortiz, R. E., Terrón-Ibarra, A. D., \& Vázquez-Carrillo, G. (2008). Evaluation of the time-cooking and tortilla making properties of quality protein maize hybrids grown in Mexico. Plant Food Human Nutrition, 63(3), 119-125. https://doi.org/10.1007/s11130-0080080-1

Singh, D., Kumar, A., Kumar, R., Kushwaha, N., Mohanty, T. A., \& Kumari, P. (2020). Genetic variability analysis of QPM (Zea mays L.) inbreds using morphological characters. International Journal Current Microbiology Applied Science, 9(2), 328-338. https://doi.org/10.20546/ijcmas.2020.902.042

Sofi, P. A., Wani, S. A., Rather, A. G., \& Wani, S. H. (2009). Review article: Quality protein maize (QPM): Genetic manipulation for the nutritional fortification of maize. Journal Plant Breeding Crop Science, 1(6), 244-253.

Ufaz, S., \& Galili, G. (2008). Improving the content of essential amino acids in crop plants: Goals and opportunities. Plant Physiology, 147, 954-961. https://doi.org/10.1104/pp.108.118091

Vargas-Escobar, E. A., Vargas-Sánchez, J. E., \& Baena-García, D. (2016). Análisis de estabilidad y adaptabilidad de híbridos de maíz de alta calidad proteica en diferentes zonas agroecológicas de Colombia. Acta Agronomica, 65(1), 72-79. https:// doi.org/10.15446/acag.v65n1.43417

Vázquez-Carrillo, M. G., Mejía-Andrade, H., Tut-Couoch, C., \& Gómez-Montiel, N. O. (2012). Características de granos y tortillas de maíces de alta calidad proteínica desarrollados para los Valles Altos Centrales de México. Revista Fitotecnia Mexicana, 35(1), 23-31.

Vivek, B. S., Krivanek, A. F., Palacios-Rojas, N., Twumasi-Afriyie, S., \& Diallo, A. O. (2008). Breeding quality protein maize $(Q P M)$ : Protocols for developing QPM cultivars. International Maize and Wheat Improvement Center.

Wegary, D., Labuschagne, M. T., \& Vivek, B. S. (2011). Protein quality and endosperm modification of quality protein maize (Zea mays L.) under two contrasting soil nitrogen environments. Field Crops Research, 121(3), 408-415. https://doi. org/10.1016/j.fcr.2011.01.010 